Anaesthesist $2022 \cdot 71: 475-482$ https://doi.org/10.1007/s00101-021-01081-9 Angenommen: 2. Dezember 2021 Online publiziert: 5 . Januar 2022

(c) Der/die Autor(en) 2022

\title{
Intensivbeatmung - neue Norm legt einheitliche Nomenklatur für Beatmungsmodi fest
}

Peter Kremeier ${ }^{1}$ - Stephan H. Böhm² · Christian Woll · Daniel A. Reuter ${ }^{2}$. Sven Pulletz ${ }^{2}$

'Simulationszentrum für klinische Beatmung, Karlsruhe, Deutschland

${ }^{2}$ Klinik und Poliklinik für Anästhesiologie und Intensivtherapie, Universitätsklinikum Rostock, Rostock, Deutschland

\section{Zusammenfassung}

Hintergrund: Die derzeitige Benennung der Beatmungsmodi in Anästhesiologie und Intensivmedizin ist geprägt von herstellerspezifischen, uneinheitlichen Akronymen. Dies ist für Anwender verwirrend und für Patienten potenziell lebensgefährlich. Mit der im August 2021 in ihrer deutschen Fassung als DIN EN ISO 19223:2021 veröffentlichten Norm soll eine einheitliche Klassifizierung mit entsprechender Nomenklatur eingeführt werden.

Ziel der Arbeit: Darstellung der neuen Norm und ihrer Konsequenzen für den Anwender.

Material und Methode: Review und Zusammenfassung der DIN EN ISO 19223:2021 mit kritischer Würdigung ihrer Stärken und Schwächen.

Ergebnisse: Ein vereinfachtes Schema zeigt die gruppenweise Klassifikation von Beatmungsmodi auf der Grundlage ähnlicher Merkmale. Diese werden durch zusätzliche Variablen weiter spezifiziert. Eine Referenztabelle stellt die neue Nomenklatur der Beatmungsmodi den aktuell gängigen gegenüber. Demnach erscheinen das neue Klassifizierungsschema uneinheitlich und die Variablen schwer zu unterscheiden.

Schlussfolgerungen: Eine genormte Terminologie und Semantik in der Beatmungsmedizin ist zur Fehlerreduzierung notwendig und erstrebenswert. Die jüngst vorgestellte Norm erfüllt diese Erwartungen jedoch nur im Ansatz und wird in ihrer jetzigen Form wahrscheinlich zu weiteren Unklarheiten und Problemen in der klinischen Routine führen. Entsprechend sollte diese erste Version der DIN EN ISO 19223:2021 zwingend als Startpunkt einer inhaltlichen Diskussion auch außerhalb der Normungsgremien verstanden werden, damit deren offensichtliche Schwächen ausgemerzt und die Nomenklatur alltagstauglich werden.

\section{Schlüsselwörter}

DIN EN ISO 19223:2021 · Beatmungsgeräte · Beatmungsformen · Beatmungsmuster · Taxonomie

\section{Kurze Hinführung zum Thema}

Die derzeitige Benennung der Beatmungsmodi in der Anästhesiologie und Intensivmedizin ist geprägt von herstellerspezifischen und somit uneinheitlichen Bezeichnungen. Dies ist nicht nur für Anwender verwirrend, sondern auch potenziell gefährlich. Eine einheitliche Klassifizierung sowie die entsprechende Nomenklatur wurde nun Ende August 2021 in der deutschen Fassung als DIN EN ISO 19223:2021 veröffentlicht. Es werden die
Zusammenhänge sowie die neue Norm zur Standardisierung der neuen Beatmungsterminologie dargestellt.

\section{Einleitung}

Die Beatmungstherapie hat sich in den letzten 70 Jahren als intensivmedizinisches Standardverfahren etabliert [1]. Parallel dazu hat sich auch die Anzahl der verfügbaren Beatmungsmodi vervielfacht (• Abb. 1). 


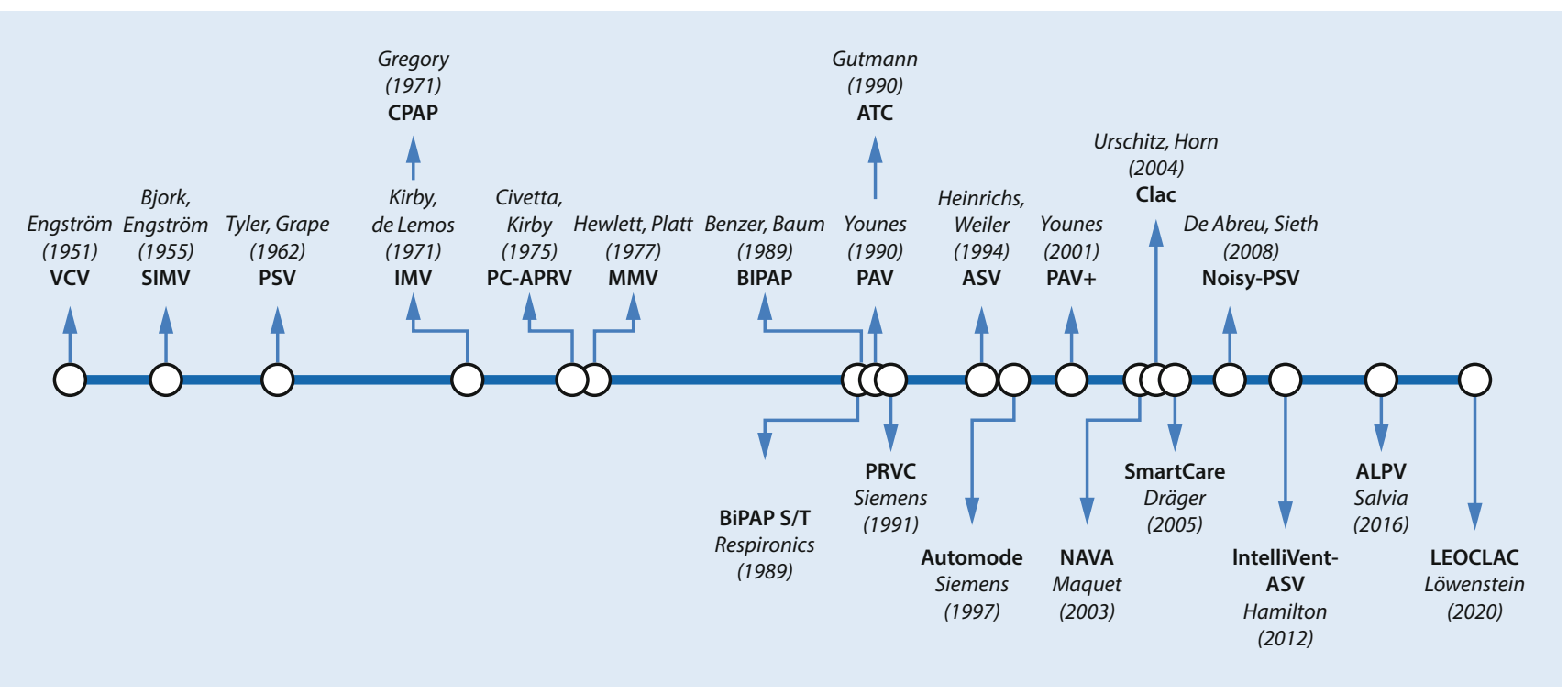

Abb. $1 \Delta$ Wichtigste Meilensteine der Entwicklung von Beatmungsmodi in den letzten 70 Jahren. (๑ All rights reserved; mit freundlicher Genehmigung von Peter Kremeier, Sven Pulletz)

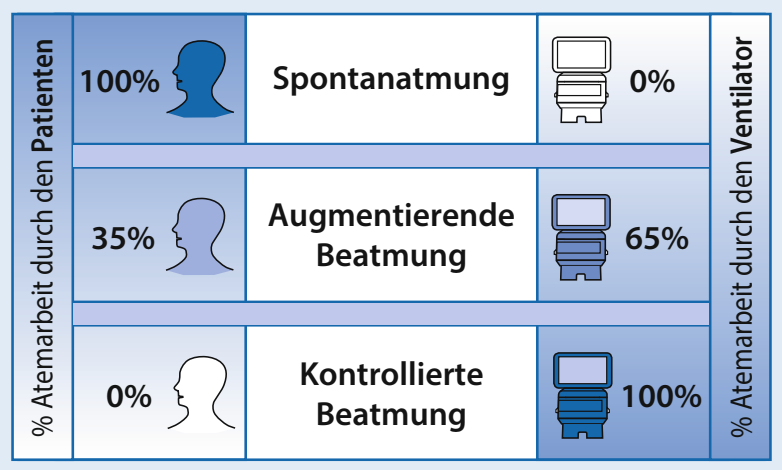

In der Anfangszeit der maschinellen Beatmung war eine sehr simple Klassifizierung aufgrund der überschaubaren Anzahl an Beatmungsmodi ausreichend, um das Verständnis der Anwender sicherzustellen. Dabei wurden Beatmungsmodi nach dem Anteil der Atemarbeit, der vom Patienten bzw. vom Beatmungsgerät übernommen wurde, unterschieden (• Abb. 2).

Allein in einem gängigen amerikanischen Lehrbuch über Beatmungsgeräte aus dem Jahre 2017 werden über 170 einzelne Beatmungsmodi beschrieben [3]. Zwar wurde dort für die Vielzahl der modernen Beatmungsmodi das Klassifizierungsschema deutlich erweitert, die Basis bildete jedoch weiterhin die einfache Klassifizierung der Modi nach der Atemarbeit in mandatorische, augmentierende und spontane.

\begin{abstract}
Abb. $2 \triangleleft$ Modifiziertes Klassifizierungsschema in Abhängigkeit von der übernommen Atemarbeit. (In Anlehnung an [2]. @ All rights reserved; mit freundlicher Genehmigung von Peter Kremeier, Sven Pulletz)
\end{abstract}

Der tatsächliche Umfang an heute verfügbaren Beatmungsmodi ist aber noch deutlich umfangreicher. Die meisten Intensivbeatmungsgeräte ermöglichen es dem Anwender, unterschiedlichste Parameter anzupassen, die den eingestellten Grundmodus umfassend modifizieren und diesen letztlich in einen anderen Modus umwandeln können. Dies geschieht vielfach unbewusst und ohne Änderung des angezeigten Beatmungsmodus. Daher gibt es erheblich mehr individuelle Beatmungsmodi (bezogen auf die Möglichkeiten der Interaktion zwischen Patienten und Beatmungsgerät), als die am Beatmungsgerät formal einzustellenden und angezeigten oder aber die in Benutzerhandbüchern und Fachbüchern genannten.

Derzeit legen Beatmungsgerätehersteller mangels gesetzlicher Regelungen oder normativer Vorgaben die Bezeichnungen und Abkürzungen ihrer Beatmungsmodi in Eigenregie fest. Dadurch gibt es eine Vielfalt an Bezeichnungen für ähnliche oder sogar identische Beatmungsmodi, die obendrein noch aus Wettbewerbs- und Marketinggründen häufig markenrechtlich geschützt werden. Der Vergleich von Beatmungsmodi verschiedener Hersteller gestaltet sich somit schwierig. Diese uneinheitlichen und herstellerspezifischen Bezeichnungen für Beatmungsmodi sind sowohl für Anwender in der klinischen Praxis als auch für Leser und Autoren von Fachbüchern und -artikeln in höchstem Maße verwirrend [4]. Das Ausmaß dieser Sprachverwirrung spiegelt die nachfolgende Synopsis in • Tab. 1 wider, die die gängigsten Beatmungsgerätehersteller und deren verwendete Abkürzungen für Beatmungsmodi im Vergleich zur DIN EN ISO 19223:2021 Norm darstellt.

Über die Jahre hinweg gab es mehrere freiwillige Initiativen zur Standardisierung der Beatmungstaxonomie. Nennenswert sind dabei insbesondere die theoretischen Überlegungen des Amerikaners Robert L. Chatburn, der 1992 erstmals ein Klassifizierungsschema für Beatmungsmodi publizierte [5]. Jedoch konnte sich Chatburns Klassifizierungsschema nicht durchsetzen und wurde von den Medizinprodukteherstellern weitgehend ignoriert. 
Tab. 1 Gegenüberstellung ausgewählter Abkürzungen verschiedener Hersteller für deren Beatmungsmodi im direkten Vergleich zum jeweiligen Vorschlag der Norm DIN EN ISO 19223:2021 (@ All rights reserved; mit freundlicher Genehmigung von Peter Kremeier, Sven Pulletz)

\begin{tabular}{|c|c|c|c|c|c|}
\hline Dräger & GE & Hamilton & Löwenstein Medical & Maquet & DIN EN ISO \\
\hline $\begin{array}{l}\text { Evita V300- } \\
\text { V800 }\end{array}$ & $\begin{array}{l}\text { Carescape R } \\
860\end{array}$ & C1-C3, C6, G5, S1 & Elisa 300-elisa 800VIT & Servo U & 19223:2021 \\
\hline $\begin{array}{l}\text { PC-CMV } \\
\text { PC-AC }\end{array}$ & - & - & $\begin{array}{l}\text { Mandatorisches BiLe- } \\
\text { veL }\end{array}$ & - & $C M V-P C$ oder $A / C-P C$ \\
\hline VC-CMV & VCV & CMV (nur C6) & VCV & VC & $C M V$-VC oder $A / C-V C$ \\
\hline VC-CMV (Pmax) & VCV (Plimit) & CMV (Flow pattern) (nur C6) & PLV & - & $\begin{array}{l}\text { CMV-VC [pLim] oder A/C-VC } \\
\text { [pLim] }\end{array}$ \\
\hline $\begin{array}{l}\text { VC-CMV- } \\
\text { Autoflow }\end{array}$ & - & $\begin{array}{l}\text { APVcmv/ } \\
(\mathrm{S}) \mathrm{CMV}+\end{array}$ & $\begin{array}{l}\text { Volumenadaptives } \\
\text { BiLevel }\end{array}$ & - & CMV-vtPC oder A/C-vtPC \\
\hline PC-CMV & PCV & PCV & PCV & PC & $C M V-P C$ oder $A / C-P C$ \\
\hline- & - & NIV-ST & PCV ST & $\begin{array}{l}\text { Automode: PC } \leftrightarrow \\
\text { PS }\end{array}$ & $S / T-P S \backslash P C(q)$ \\
\hline- & - & - & BiLevel ST & - & $S / T-P S \backslash P C$ \\
\hline- & - & - & $\begin{array}{l}\text { Dynamisches BiLevel- } \\
\text { ST }\end{array}$ & - & S/T-PSIvtPC \\
\hline- & - & - & Duales BiLevel-ST & - & $S / T$-vtPSIvtPC \\
\hline PC-BIPAP & BiLevel & DuoPAP & BiLevel & BiVent & SIMV-PCIPS \\
\hline $\begin{array}{l}\text { VC-SIMV- } \\
\text { Autoflow }\end{array}$ & PC-SIMV VG & $\begin{array}{l}\text { APVsimv } \\
\text { SIMV+ }\end{array}$ & Dynamisches BiLevel & PRVC & SIMV-vtPCIPS \\
\hline- & - & - & Duales BiLevel & - & SIMV-vtPClvtPS \\
\hline PC-MMV & - & - & Optionales BiLevel & - & $M M V-P C \backslash P S$ \\
\hline- & - & - & Flexibles BiLevel & - & $M M V$-vtPClvtPS \\
\hline PC-SIMV & SIMV-PC & P-SIMV+ (nur C6) & PC-SIMV & SIMV-PC & SIMV-PC\PS \\
\hline VC-SIMV & SIMV-VC & SIMV (nur C6) & VC-SIMV & SIMV-VC & SIMV-VCIPS \\
\hline VC-MMV & - & - & Optionales VCV & - & $M M V-V C \backslash P S$ \\
\hline- & - & - & Flexibles VCV & - & $M M V-V C l v t P S$ \\
\hline PC-APRV & APRV & APRV & PC-APRV & BiVent-APRV & $I M V-P C$ oder IMV-PC\{S\} \\
\hline- & - & ASV & WOBOV & - & $M M V-v t P C \backslash P S$ \\
\hline- & - & ASV 1.1 & ALPV & - & $M M V-v t P C \mid v t P S$ \\
\hline- & - & INTELLiVENT-ASV & - & - & $M M V-v t P C \backslash P S$ \\
\hline SPN-CPAP/PS & CPAP/PSV & Spont & PSV & PS & CSV-PS \\
\hline SPN-CPAP/VS & VS & VS & Dynamisches PSV & VS & CSV-vtPS \\
\hline SPN-PPS & - & - & Proportionales PSV & - & $C S V-E S[q+v]$ \\
\hline SPN-CPAP & CPAP & Spont & CPAP & Spont./CPAP & CPAP oder CSV-ACAP \\
\hline- & - & - & PAPS & - & $C S V-E S[q+v]$ \\
\hline- & - & - & - & NAVA & CSV-ES[EMG] \\
\hline
\end{tabular}

Auch die Autoren der S3-Leitlinie "Invasive Beatmung und Einsatz extrakorporaler Verfahren bei akuter respiratorischer Insuffizienz" sahen sich mit dem Problem der fehlenden Einheitlichkeit der Klassifizierung von Beatmungsmodi konfrontiert [6]. Zwar wird in dieser S3-Leitlinie auch auf das Chatburn-Klassifizierungsschema eingegangen, die Autoren der Leitlinie kamen allerdings zu dem Schluss, dass „von dieser technischen Kategorisierung in Teilen" abgewichen und ein "Kompromiss zwischen den objektiv technischen Kriterien und einer klinisch sinnvollen Einteilung der Beatmungsverfahren" gefunden wer- den müsse [6]. Entsprechend wurde für die Unterkategorien ein neues Klassifizierungsschema eingeführt.

Zur Beseitigung dieses auch sicherheitsrelevanten Problembereichs tagte erstmals 2013 das Unterkomitee SC3 der Internationalen Kommission für Normung zum Thema „Lung ventilators and related equipment". Normen spiegeln den Stand der Technik wider, der im Zuge der Zulassung als Medizinprodukt nachgewiesen werden muss. Deren Anwendung kann freiwillig erfolgen oder auf Grundlage von nationalen oder europäischen Vorgaben verpflichtend festgeschrieben sein. Die
Internationale Organisation für Normung (ISO) ist die internationale Vereinigung von Normungsorganisationen und erarbeitet internationale Normen. Das Deutsche Institut für Normung (DIN) erstellt nationale Normen, vertritt aber auch die deutschen Interessen auf ISO-Ebene. Weiterhin arbeitet die ISO mit dem Europäischen Komitee für Normung (CEN) zusammen, das als europäische Normungsorganisation einen Großteil der ISO-Normen in das europäische Normensystem überführt. Während die Überführung von ISO-Normen in das jeweilige nationale Normungsgefüge freiwillig ist, müssen sämtliche europäischen 
Normen von den CEN-Mitgliedern als nationale Norm implementiert werden. Es ist offen, wann die neue DIN ISO 19223:2021 verpflichtend eingeführt werden muss und damit fester Bestandteil eines jeden neuen Medizinproduktes oder gar jedes neuen Softwarestandes eines Medizinproduktes werden wird. Die Ziele des Normgebers waren die Entwicklung einer gemeinsamen Sichtweise sowie die Schaffung rationaler Grundlagen für eine kohärente Terminologie zur Beschreibung der Funktion von Beatmungsgeräten [7]. Die in der Norm festgelegte Terminologie und Semantik sollen ihren Weg in wissenschaftliche Veröffentlichungen, Lehrbücher und Schulungen finden und so im Verlauf der Zeit eine genormte grundlegende Sprache der maschinellen Beatmung international begründen [7]. Das Dokument postuliert als Hauptziel „soweit wie möglich bestehende Begriffe zu verwenden, ihre Bedeutung zu klären und das Potenzial einer Fehlanwendung durch genauere Festlegung zu begrenzen" [7]. Hierdurch soll der "geschulte Bediener in die Lage versetzt werden, problemlos zwischen verschiedenen Beatmungsgeräten zu wechseln und jedes Gerät nach wenigen Minuten Schulung sicher zu bedienen" [7].

\section{Beatmungsmodi - auf dem Weg zu einer einheitlichen Klassifizierung}

In der neuen Norm DIN EN ISO 19223:2021 werden Beatmungsmodi in vier Schritten klassifiziert. In einem ersten Schritt wird anhand deren Charakteristik eine Vorsilbe (Präfix) ausgewählt, die der normkonformen Bezeichnung vorangestellt wird. Für jedem Präfix sind Definitionen hinterlegt. Alle rein kontrollierten Beatmungsmodi werden demnach mit dem Präfix "CMV" ("continuous mandatory ventilation“) versehen (• Abb. 3 und 4). Bei der ausschließlich kontrollierten Beatmung übernimmt das Beatmungsgerät die gesamte Ventilation, während der Patient keinerlei Atemarbeit erbringt und somit auch nicht triggert. Der synonyme Begriff in der Literatur lautet vielfach mandatorische Beatmung. Wobei mandatorisch bedeutet, dass die Vorgabe der notwendigen Parameter im Mandat des Bedieners liegt [8]. Die Norm führt aus, dass ein kontrollierter Atemzug immer durch das Beatmungsgerät ausgelöst und in Abwesenheit von Spontanatmungsaktivität auch vollständig durch den eingestellten oder durch den vom Bediener ausgewählten Inflationstyp generiert wird. Die Klassifizierung mit dem Präfix „IMV“ (,intermittent mandatory ventilation") erfolgt nur, wenn die mandatorisch eingestellte Atemfrequenz nicht durch Trigger-Aktivitäten synchronisiert oder erhöht werden kann, gleichzeitig jedoch uneingeschränkte Atemaktivitäten beispielsweise in den Exspirationsphasen möglich sind. In der klinischen Praxis sind Beatmungsmodi ohne Trigger-Option für die mandatorischen Anteile eher selten anzutreffen. Können mandatorische Beatmungszüge getriggert werden, so wird der Modus mit der Vorsilbe " $\mathrm{A} / \mathrm{C}^{\prime}$ (, assist/control ventilation“) versehen. Im Unterschied hierzu kann der Patient bei Modi mit dem Präfix "SIMV“ (,synchronized intermittend mandatory ") durch erfolgreiche Triggerung die eingestellten mandatorischen Atemzüge auslösen und dazwischen frei spontan atmen. Hierzu kann beispielsweise eine Druckunterstützung eingestellt werden (• Abb. 3). Eine Vielzahl von modernen Beatmungsmodi basiert nicht auf einer Kombination von fest eingestellten mandatorischen Atemfrequenzen und den dazwischen liegenden Spontanatemphasen. Maschinelle Beatmungszüge werden im Bedarfsfall nur dann appliziert, wenn in einem definierten Zeitfenster das vorgewählte Mindestspontanatemminutenvolumen unterschritten wird, und reichen von einer punktuellen Ergänzung der Spontanatmung bis hin zu einer vollständigen Übernahme der Ventilation. Diese Modi werden dann mit der Vorsilbe "MMV“ ("minimum minute volume") versehen. Die nicht nur bei nichtinvasiven oder außerklinischen Beatmungsgeräten häufig anzutreffen ST-Modi, eine Kombination aus reiner Spontanatmung mit einer vorgewählten Mindestatemfrequenz pro Minute, werden mit dem Präfix "S/T" („spontaneous/timed“) versehen. Im Gegensatz zu den als SIMV klassifizierten Modi wählen ",S/T"-Modi einen alternativen Algorithmus zur fakultativen Auslösung mandatorischer Atemzügen. Bleibt nämlich in der eingestellten Zykluszeit (=60 s geteilt durch die Atemfrequenz) ein spontaner Atemzug aus, so wird dieser durch einen mandatorischen ersetzt.

In einem zweiten Schritt wird bei allen Modi mit mandatorischen Anteilen die Art des „Inflationstyps" angegeben. Unter Inflationstypen versteht die Norm die einstellbaren Zielgrößen des Atemzuges, der entweder volumenkontrolliert („volume controlled, VC"), druckkontrolliert („pressure controlled, PC") oder aber auch druckkontrolliert mit Volumengarantie (,vtPC ) verabreicht werden kann. In der Literatur wird diese Zielgröße auch oft als Kontrollvariable bezeichnet, die durch den Anwender eingestellt und während der Inspiration seitens des Gerätes automatisch geregelt und damit kontrolliert wird.

Im dritten Schritt wird die Art der Spontanatmung klassifiziert. Reine Spontanatmungsformen erhalten das Präfix "CSV" ("Continuous spontaneous ventilation“). Anhand der Charakteristik der Druckunterstützung wird nun die Art der Spontanatmung beschrieben. Entsprechend stehen "CSV-PS" („pressure support ${ }^{\prime \prime}$ ) für die fest vorgegebene Druckunterstützung, "CSV-vtPS“ („tidal volume") für die Druckunterstützung mit Volumengarantie und "CSV-ES" („effort support") für alle Arten der proportionalen Druckunterstützung. Die Kombination aus Tubuskompensation und Spontanatmung auf CPAP-Niveau wird als "CSV-TC" ("tube compensation“) klassifiziert. Eine Ausnahme bei den Modi mit reiner Spontanatmung bildet die CPAP-Beatmung ohne Druckunterstützung, die unverändert als "CPAP" ("continuous positive airway pressure") bezeichnet wird. Diese wird alternativ auch als „ACAP“ (,assured constant airway pressure") bezeichnet, wenn „der Atemwegsdruck ungeachtet der Inspirations- oder Exspirationsdurchflüsse oder der festgelegten zulässigen Leckage bei seinem eingestellten Wert gehalten wird, wann immer dieser auf einem konstanten Niveau bleiben soll“ [7]. In einem letzten Schritt können noch spezielle Varianten der Beatmung durch Hinzufügung eines nachgestellten Codes beschrieben werden. Beispielsweise weist ein kleines $v$ in runden Klammern als "(v)" darauf hin, dass die Inspirationsphase eines druckkontrollierten Modus durch das Erreichen eines vorgegebenen 


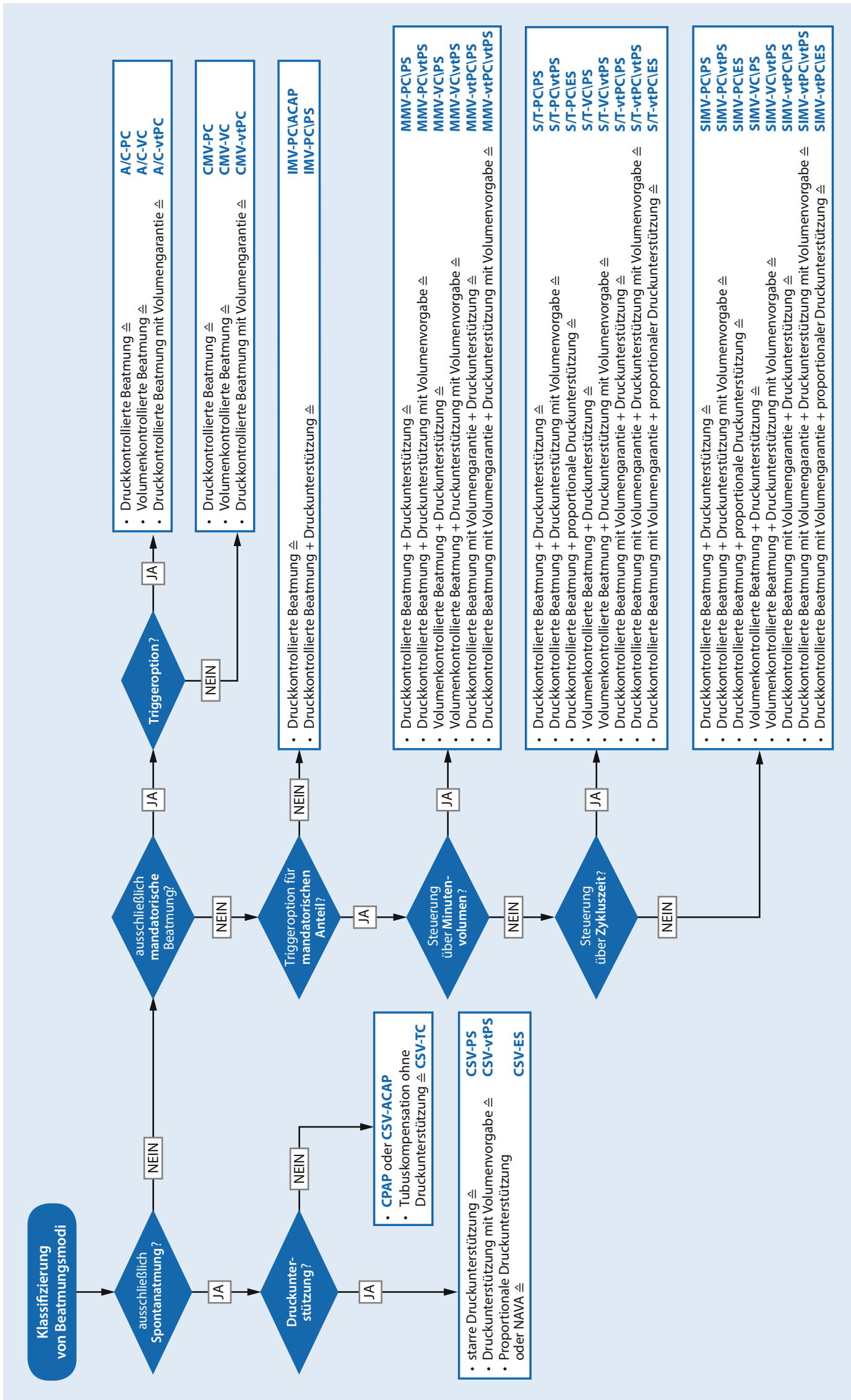

Abb. $3 \triangleleft$ Flussdiagramm der Klassifizierung der verschiedenen Beatmungsmodi gemäß DIN ISO 19223:2021. Die als Raute dargestellten Entscheidungskriterien zeigen an, wie die einzelnen Beatmungsmodi zu verschiedenen Gruppen zusammengefasst werden. Die Nomenklatur richtet sich nach der Einordnung in die entsprechende Gruppe. Die entsprechenden Abkürzungen, aus denen sich die Nomenklatur zusammensetzt, sind in 0 Abb. 4 dargestellt. ( $\odot$ All rights reserved; mit freundlicher Genehmigung von Peter Kremeier, Sven Pulletz) 


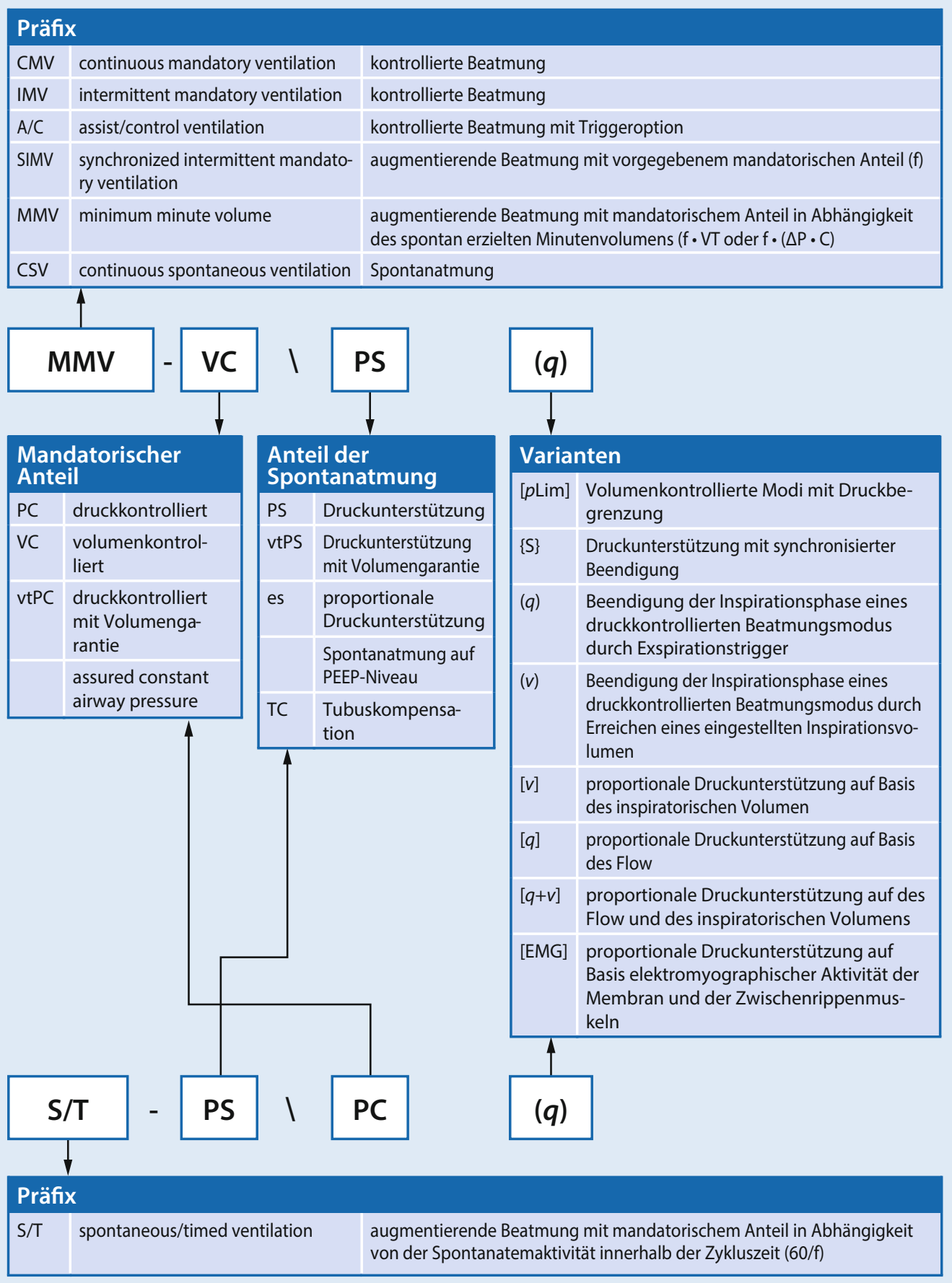

Abb. $4<$ Aufbau der Nomenklatur der einzelnen Beatmungsmodi gemäß DIN EN ISO 19223:2021 (৫) All rights reserved; mit freundlicherGenehmigung von Peter Kremeier, Sven Pulletz)
Inspirationsvolumen beendet wird [7]. Demgegenüber kennzeichnet ein kleines $v$ in eckigen Klammern als "[v]" eine proportionale Druckunterstützung auf Basis eines inspiratorischen Tidalvolumens [7]. Der Form der Klammer kommt also bei der eindeutigen Zuordnung der Varianten eine entscheidende und gleichzeitig verwirrende Bedeutung zu. In der klinischen Anwendung wird die Unterscheidung verschiedener Beatmungsmodi anhand der unterschiedlichen Formen der Klammern für erhebliche Probleme sorgen. Ebenso schwer zu durchschauen ist die uneinheitliche Position der Inflationstypen innerhalb der zuvor beschriebenen taxonomischen Sequenz bei der Darstellung der Anteile der mandatorischen und spontanen Atmung, die bei unterschiedlichen Beatmungsmodi getauscht wird.
Dies wird beispielhaft in 0 Abb. 4 gezeigt. Die Umstellung auf die neue Norm wird für erfahrene Nutzer auch dadurch noch einmal anspruchsvoller, dass Abkürzungen aus alten Benennungen mit neuen erweiterten Bedeutungen belegt werden. So schlägt die Norm etwa vor, das Präfix SIMV, das bisher ausschließlich für eine synchronisierte volumenkontrollierte Beatmungsform stand, fortan auch 
für druckkontrollierte Beatmungsmodi zu verwenden.

Ein weiterer hochgradig problematischer Punkt ist die schiere Länge der teils kryptischen Abkürzungen der Beatmungsmodi. Diese neue Taxonomie, die z.T. 12 und mehr Zeichen verwendet, wird erheblich schwerer auf dem Display von Beatmungsgeräten in übersichtlicher Form unterzubringen sein.

\section{Gruppenweise Klassifikation von Beatmungsmodi auf der Grundlage ähnlicher Merkmale - Vorgehensweise und Grenzen}

Alle bisherigen Klassifizierungsversuche, der neue Normentwurf 19223:2021 eingeschlossen, stoßen bei der tatsächlichen Komplexität der Beatmungsmodi schnell an ihre Grenzen. Vielfach sind diese eher technisch geprägt und orientieren sich weniger an klinischen Fragestellungen. Deshalb versucht die gruppenweise Klassifikation, Beatmungsmodi auf der Grundlage ähnlicher Merkmale zu klassifizieren. Im klinischen Alltag sind diese ähnlichen Merkmale vielfach bedeutsam für das Verständnis sowie die Gestaltung der Beatmungstherapie. Am Beispiel der proportionalen Druckunterstützungen und der "neurally adjusted ventilatory assist" (NAVA) lässt sich exemplarisch sowohl die europäische Klassifizierungsstrategie als auch die Kritik in Fachkreisen an dieser ersten Version der Norm aufzeigen. Bei der proportionalen Druckunterstützung wird nicht die Höhe der Druckunterstützung konstant gehalten, sondern das Verhältnis zwischen Druckunterstützung und Atemanstrengung. Die geräteseitige erzeugte Druckunterstützung ist über die gesamten Einatmungsbemühungen des Patienten proportional zu der vom Patienten geleisteten Atemarbeit [9]. Je nach Art des Modus kann der Umfang der notwendigen Druckunterstützung mit einer regelmäßigen endinspiratorischen Ermittlung der Atemarbeit individuell an die Atemarbeit des Patienten angepasst werden [10]. Die erhobenen Werte erlauben es, fortlaufend die Relation zwischen Druckunterstützung und Atemanstrengung zu ermitteln und selektiv zu kompensieren. Ein anderer Weg zur proportionalen Unterstützung wird mit NAVA verfolgt. Die Synchronisa- tion der inspiratorischen Unterstützung erfolgt proportional zum nervalen Aktivierungsimpuls für die Zwerchfellkontraktion. Dieser wird über eine spezielle Magensonde mittels ösophagealen Elektroden ermittelt. Basierend auf dem Erregungspotential der zuleitenden Nerven wird letztlich der notwendige Unterstützungsgrad realisiert [11]. Gemäß neuer 19233-Norm werden alle proportionalen Druckunterstützungen in einer Gruppe mit ähnlichen Merkmalen zusammengefasst und als „Continuous spontaneous ventilation effort-support (CSV-ES)“ klassifiziert [7]. Spezielle Variantenbezeichnungen sollen dann aber dann doch die z. T. erheblichen Unterschiede zwischen den Modi dieser Gruppe zumindest teilweise abbilden. Entsprechend weist die Norm „CSV-ES $[q+v]]^{\prime}$ als proportionale Druckunterstützung auf Basis eines Inspirationsflow und eines inspiratorischen Volumens aus, wohingegen „CSV-ES [EMG]" für die NAVA-basierte Druckunterstützung. Die Verwirrung wird durch den Zusatz "[EMG]" komplett, da bei NAVA nicht etwa ein EMG, sondern vielmehr die nervale Aktivität der Nn. phrenici gemessen wird. Den z.T. fundamentalen Unterschieden der Ansätze zu Einstellung bzw. Anpassung der hier vorstellten proportionalen Druckunterstützung mit oder ohne eine atemzugsweise Messung der Atemarbeit wird die gemeinsame neue Bezeichnung CSV-ES trotz Ergänzung der Varianten nicht gerecht.

Die Limitation der gruppenweisen Klassifizierung auf der Grundlage ähnlicher Merkmale zeigt sich gerade bei komplexeren Beatmungsmodi, die mit erweiterten Messverfahren kombiniert werden oder mit künstlicher Intelligenz versehen sind. Zwar war das Ziel der Normgeber mittels definierten Vokabulars dem Anwender eine klare Vorstellung davon zu vermitteln, „wie die gewählten Einstellungen die Wechselwirkung zwischen dem Patienten und dem Beatmungsgerät beeinflussen würden" [7]. Doch dieser Anspruch wurde leider vielfach nicht erfüllt. Eine klinisch sinnvolle Klassifizierung von Beatmungsmodi muss jedoch für den klinischen Nutzer so transparent sein, dass die Funktionsweisen sofort erkannt, die Folgen für den Patienten konkret eingeschätzt und notwendige Einstellungen folgerichtig gewählt werden können.

\section{Fazit für die Praxis}

Unbestritten sind eine genormte Terminologie und Semantik in der Beatmungsmedizin im Hinblick auf die Fehlerreduzierung notwendig und erstrebenswert. Mit der vorliegenden Norm hat man versucht, sich des Problems der herstellerseitigen individuellen Benennung von Beatmungsmodi und der damit verbunden Verwirrung anzunehmen. Dies ist mit der aktuellen Version der Norm leider nicht gelungen. Vielmehr wird diese neue Norm wahrscheinlich zu weiteren neuen Unklarheiten und Problemen in der klinischen Routine führen. Probleme bei der eindeutigen Klassifizierung der Beatmungsmodi, neu eingeführte Begrifflichkeiten, aber auch neue Bedeutungen für altbewährte Begrifflichkeiten sowie die problematische Kennzeichnung der Varianten sind einem besseren Verständnis abträglich. Entsprechend muss die erste Version der DIN EN ISO 19223:2021 zwingend als Startpunkt einer inhaltlichen Diskussion auch außerhalb der Normungsgremien verstanden werden, damit die Schwächen des Entwurfes ausgemerzt und die Ergebnisse alltagstauglich werden.

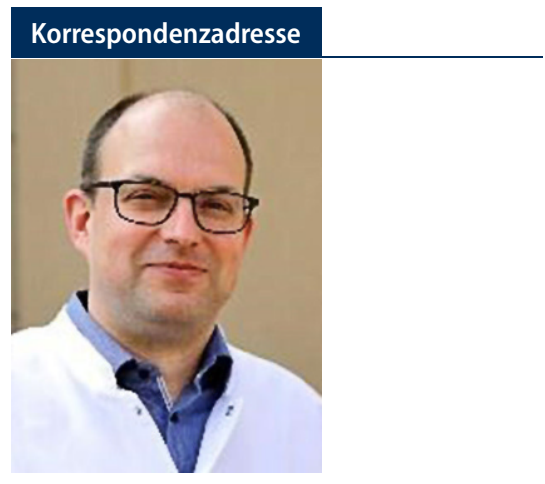

Dr. Sven Pulletz

Klinik und Poliklinik für Anästhesiologie und Intensivtherapie, Universitätsklinikum Rostock Schillingallee 35, 18057 Rostock, Deutschland sven.pulletz@med.uni-rostock.de

Funding. Open Access funding enabled and organized by Projekt DEAL.

\section{Einhaltung ethischer Richtlinien}

Interessenkonflikt. P. Kremeier gibt an, Beratungsgebühren von Löwenstein medical Innovation $\mathrm{GmbH}$ \& Co KG (vormals Salviamedical Innovation GmbH \& Co KG) und Löwenstein medical GmbH \& Co KG erhalten zu haben. Er erhält Bezahlung als Leiter des Simulationszentrum für Intensivbeatmung der Meducation Service Akademie GmbH. S.H. Böhm, C. Woll, D. A. Reuter und S.Pulletz geben an, dass kein Interessenkonflikt besteht. 
Für diesen Beitrag wurden von den Autoren keine Studien an Menschen oder Tieren durchgeführt. Für die aufgeführten Studien gelten die jeweils dort angegebenen ethischen Richtlinien.

Open Access. Dieser Artikel wird unter der Creative Commons Namensnennung 4.0 International Lizenz veröffentlicht, welche die Nutzung, Vervielfältigung, Bearbeitung, Verbreitung und Wiedergabe in jeglichem Medium und Format erlaubt, sofern Sie den/die ursprünglichen Autor(en) und die Quelle ordnungsgemäß nennen, einen Link zur Creative Commons Lizenz beifügen und angeben, ob Änderungen vorgenommen wurden.

Die in diesem Artikel enthaltenen Bilder und sonstiges Drittmaterial unterliegen ebenfalls der genannten Creative Commons Lizenz, sofern sich aus der Abbildungslegende nichts anderes ergibt. Sofern das betreffende Material nicht unter der genannten Creative Commons Lizenz steht und die betreffende Handlung nicht nach gesetzlichen Vorschriften erlaubt ist, ist für die oben aufgeführten Weiterverwendungen des Materials die Einwilligung des jeweiligen Rechteinhabers einzuholen.

Weitere Details zur Lizenz entnehmen Sie bitte der Lizenzinformation auf http://creativecommons.org/ licenses/by/4.0/deed.de.

\section{Literatur}

1. Statistisches Bundesamt (2019) Fallpauschalenbezogene Krankenhausstatistik (DRG-Statistik) Operationen und Prozeduren der vollstationären Patientinnen und Patienten in Krankenhäusern bis zum kodierbaren Endpunkt

2. Oczenski, W. (2017). Atmen, Atemhilfen. Stuttgart

3. Cairo JM (Hrsg) (2017) Mosby's respiratory care equipment, 10. Aufl. Elsevier, Amsterdam

4. Rabec C, Langevin B, Rodenstein D et al (2012) Ventilatory modes. What's in a name? Respir Care 57(12):2138-2139

5. Branson RD, Chatburn RL (1992) Technical description and classification of modes of ventilator operation. Respir Care 37(9):1026-1044

6. Adamzik M, Bauer A, Bein T, Deja M, Dembinski R, Ellger B, Fichtner F, Gama de Abreu M, Kaisers S (2017) S3-Leitlinie Invasive Beatmung und Einsatz extrakorporaler Verfahren bei akuter respiratorischer Insuffizienz

7. Deutsches Institut für Normung e.V. (2021) Beatmungsgeräte und zugehörige Geräte Terminologie und Semantik (ISO 19223:2019 EN ISO 19223:2021)

8. Rathgeber J (2010) Grundlagen der maschinellen Beatmung. Thieme, Stuttgart

9. Haberthür C, Guttmann J, Osswald PM et al (2001) Beatmungskurven. Kursbuch und Atlas. Springer, Berlin, Heidelberg

10. Younes M, Webster K, Kun J et al (2001) A method for measuring passive elastance during proportional assist ventilation. Am J Respir Crit Care Med 164(1):50-60. https://doi.org/10.1164/ ajrccm.164.1.2010068

11. Colombo D, Cammarota G, Bergamaschi V et al (2008) Physiologic response to varying levels of pressure support and neurally adjusted ventilatory assist in patients with acute respiratory failure. Intensive Care Med 34(11):2010-2018. https://doi. org/10.1007/s00134-008-1208-3

\section{Intensive Care ventilation-New norm establishes a uniform nomenclature for ventilation modes}

Background: The current naming of ventilation modes in anesthesiology and critical care is characterized by manufacturer-specific inconsistent acronyms. This is confusing for users and potentially life-threatening for patients. The standard, published in August 2021 in its German version as DIN EN ISO 19223:2021, aims to introduce a uniform classification with corresponding nomenclature.

Aim of the work: To present the new standard and its consequences for the user. Material and method: Review and summary of DIN EN ISO 19223:2021 with a critical appraisal of its strengths and weaknesses.

Results: A simplified scheme shows the group classification of ventilation modes based on similar characteristics. These are further specified by additional variables. A reference table contrasts the new nomenclature of ventilation modes with those currently in use. Accordingly, the new classification scheme appears inconsistent and the variables are difficult to distinguish.

Conclusion: Standardized terminology and semantics in respiratory care are necessary and desirable for error reduction. However, the recently presented standard fulfils these expectations only to some extent and in its current form will probably lead to further ambiguities and problems in the clinical routine. Accordingly, it is imperative that this first version of DIN EN ISO 19223:2021 be understood as the starting point for a discussion of its content, even outside the standards committees, so that its obvious weaknesses can be eradicated and the nomenclature made suitable for everyday use.

\section{Keywords}

DIN EN ISO 19223:2021 · Ventilators · Ventilation modes · Ventilation patterns · Taxonomy 